\title{
Correction to: Multicenter phase II study of trastuzumab plus S-1 alone in elderly patients with HER2-positive advanced gastric cancer (JACCRO GC-06)
}

\author{
Yutaka Kimura ${ }^{1} \cdot$ Masashi Fujii $^{2} \cdot$ Toshiki Masuishi $^{3} \cdot$ Kazuhiro Nishikawa $^{4} \cdot$ Chikara Kunisaki $^{5} \cdot$ Satoshi Matsusaka $^{6}$. \\ Yoshihiko Segawa ${ }^{7} \cdot$ Masato Nakamura $^{8} \cdot$ Kinro Sasaki $^{9} \cdot$ Narutoshi Nagao $^{10}$. Yukimasa Hatachi ${ }^{11}$. Yasuhiro Yuasa ${ }^{12}$. \\ Shinya Asami ${ }^{13} \cdot$ Masahiro Takeuchi $^{14} \cdot$ Hiroshi Furukawa $^{1} \cdot$ Toshifusa Nakajima $^{2}$. on behalf of the JACCRO GC-06 \\ Study Group
}

Published online: 12 December 2017

(c) The International Gastric Cancer Association and The Japanese Gastric Cancer Association 2017

\section{Correction to: Gastric Cancer https://doi.org.10.1007/s10120-017-0766-x}

The correct name of the twelfth author should be "Yasuhiro Yuasa", and not "Yasuhiko Yuasa" as given in the original publication of the article.

The original article was corrected.

The original article can be found online at https://doi. org. 10.1007/s10120-017-0766-x.

Masashi Fujii

gioncology2006@yahoo.co.jp

1 Department of Surgery, Kindai University Faculty of Medicine, Osaka-Sayama, Japan

2 Japan Clinical Cancer Research Organization, 7F Ginza Wing Building, 1-14-5, Ginza, Chuo-ku, Tokyo 104-0061, Japan

3 Department of Clinical Oncology, Aichi Cancer Center Hospital, Nagoya, Japan

4 Department of Surgery, Osaka National Hospital, Osaka, Japan

5 Department of Surgery, Gastroenterological Center, Yokohama City University, Yokohama, Japan

6 Department of Gastroenterology, Cancer Institute Hospital of the Japanese Foundation for Cancer Research, Tokyo, Japan
7 Department of Medical Oncology, Saitama Medical University International Medical Center, Hidaka, Japan

8 Aizawa Comprehensive Cancer Center, Aizawa Hospital, Matsumoto, Japan

9 First Department of Surgery, Dokkyo Medical University Hospital, Tochigi, Japan

10 Department of Surgery, Gifu Prefectural General Medical Center, Gifu, Japan

11 Department of Medical Oncology, Kobe City Medical Center General Hospital, Kobe, Japan

12 Department of Surgery, Tokushima Red Cross Hospital, Tokushima, Japan

13 Department of Surgery, Fukuyama City Hospital, Fukuyama, Japan

14 Department of Clinical Medicine (Biostatistics), Kitasato University School of Pharmacy, Tokyo, Japan 